\title{
Effects of Vedolizumab Therapy on Extraintestinal Manifestations in Inflammatory Bowel Disease
}

\author{
Mark Fleisher $^{1} \cdot$ Jan Marsal ${ }^{2} \cdot$ Scott D. Lee $^{3} \cdot$ Laura E. Frado $^{4} \cdot$ Alyssa Parian $^{5} \cdot$ Burton I. Korelitz $^{6} \cdot$ Brian G. Feagan $^{7}$
}

Received: 3 October 2017 / Accepted: 8 February 2018 / Published online: 26 February 2018

(C) The Author(s) 2018. This article is an open access publication

\begin{abstract}
Background Approximately 15-20\% of ulcerative colitis patients and 20-40\% of those with Crohn's disease experience extraintestinal manifestations (EIMs) of their inflammatory bowel disease (IBD). Clinicians who treat IBD must manage EIMs affecting multiple organs that variably correlate with intestinal disease activity. Vedolizumab is a monoclonal antibody for the treatment of IBD with a gut-selective mechanism of action.

Aims This report evaluates whether vedolizumab is an effective treatment of EIMs, given its gut-specific mechanism of action.

Methods We report 8 case studies of patients with various EIMs, including pyoderma gangrenosum, peripheral arthralgia/ arthritis, axial arthropathies, erythema nodosum, and uveitis, who received vedolizumab therapy.

Results Vedolizumab therapy was effective for pyoderma gangrenosum in ulcerative colitis, uveitis, erythema nodosum, polyarticular arthropathy, and ankylosing spondylitis/sacroiliitis but did not provide sustained benefit for the treatment of pyoderma gangrenosum in a patient with Crohn's disease.
\end{abstract}

Conclusions These cases demonstrate the potential of vedolizumab as a treatment of EIMs in patients with IBD.

Keywords Inflammatory bowel disease $\cdot$ Crohn's disease $\cdot$ Ulcerative colitis $\cdot$ Extraintestinal manifestations

\section{Introduction}

Inflammatory bowel disease (IBD), which comprises ulcerative colitis (UC) and Crohn's disease (CD), is characterized by chronic intestinal inflammation that results in mucosal ulceration and tissue damage [1]. Although the etiology of IBD is not yet fully elucidated, multiple factors including genetic susceptibility, environmental influences, the intestinal microbiota, and defective host defense mechanisms contribute to the pathological process [2]. Failure to adequately control inflammation frequently leads to bowel damage and disability [3], and many patients will eventually require surgery [4].

\author{
Borland-Groover Clinic, Jacksonville, FL, USA \\ Lund University, Lund, Sweden \\ University of Washington, Seattle, WA, USA \\ 4 Mount Sinai Hospital, New York, NY, USA \\ 5 Johns Hopkins Hospital, Baltimore, MD, USA \\ 6 Division of Gastroenterology, Department of Medicine, \\ Lenox Hill Hospital, New York, NY 10075, USA \\ 7 Robarts Clinical Trials, Robarts Research Institute, \\ Western University, 100 Dundas Street, Suite 200, London, \\ ON N6A 5B6, Canada
}


Early introduction of highly effective anti-inflammatory therapy has been shown to prevent IBD-related complications in both UC and CD [5]. In CD, treatment with tumor necrosis factor (TNF) antagonists has demonstrated increased rates of mucosal healing and clinical remission [6-8] and decreased rates of hospitalization and surgery [3]. Similar results have been obtained with infliximab therapy in UC. These findings have led to a fundamental change in the treatment paradigm for both diseases such that the recent STRIDE (Selecting Therapeutic Targets in Inflammatory Bowel Disease) guidelines have incorporated endoscopy, an objective marker of inflammation, as a treatment target in both conditions [9].

\section{Extraintestinal Manifestations}

Despite this nascent emphasis on controlling inflammation, little attention has been paid to the treatment of extraintestinal manifestations (EIMs) in IBD. Prevalence estimates currently suggest that EIMs are present in 15-20\% of UC patients and $20-40 \%$ of CD patients [10]. EIMs adversely affect quality of life, and some EIMs, such as primary sclerosing cholangitis or venous thromboembolism, can be lifethreatening [11].

Currently, the pathophysiology of EIMs is poorly understood; however, possible mechanisms include antigen leakage from the gut resulting in immune complex-mediated small vessel vasculitis and molecular mimicry, whereby shared epitopes between intestinal bacteria and antigens of the joints and skin trigger immune responses at extraintestinal sites [12]. Although the relationship between EIMs and intestinal disease activity varies depending on the type of EIM, in general, effective treatment of the local intestinal inflammation should control many EIMs of IBD.

The musculoskeletal, dermatologic, ocular, hepatopancreatobiliary, renal, and hematologic systems are common organ systems affected (Table 1); and of those, musculoskeletal EIMs are the most common [13]. Peripheral arthralgia/arthritis, which occurs with an estimated prevalence of 5-20\% [14], can be classified as type 1 (pauciarticular) or type 2 (polyarticular). Type 1 disease mainly affects large joints, including the knees, ankles, wrists, and elbows, and usually correlates with IBD activity [14]. Type 2 mainly affects small joints with a symmetrical distribution, including the metacarpophalangeal and interphalangeal joints, and generally does not correlate with luminal inflammation [14].

Axial arthropathies, a second type of musculoskeletal EIM, have a prevalence between 5 and 12\% (Table 1) [15]. Axial arthropathies include ankylosing spondylitis, a systemic inflammatory disease that affects the sacroiliac joints, the spine, and potentially other joints, but may also present as isolated sacroiliitis. Both versions usually do not correlate with intestinal IBD activity [16]. TNF antagonists are highly effective treatment for ankylosing spondylitis [17]. Although the European Crohn's and Colitis Organization (ECCO) currently recommends that TNF antagonist therapy be used early in IBD patients with axial arthritis

Table 1 Common extraintestinal manifestations

\begin{tabular}{|c|c|c|c|c|}
\hline Site & Prevalence & Manifestation & & $\begin{array}{l}\text { Correlates with } \\
\text { bowel inflamma- } \\
\text { tion? }\end{array}$ \\
\hline \multirow[t]{4}{*}{ Musculoskeletal } & \multirow[t]{2}{*}{$5-20 \%[14]$} & \multirow[t]{2}{*}{ Peripheral arthralgia/arthritis } & Type 1 (large joints) & Yes [14] \\
\hline & & & Type 2 (small joints) & No [14] \\
\hline & \multirow[t]{2}{*}{$5-12 \%[15]$} & \multirow[t]{2}{*}{ Axial arthropathies } & Ankylosing spondylitis & No [16] \\
\hline & & & Isolated sacroiliitis & No [16] \\
\hline \multirow[t]{3}{*}{ Dermatologic } & \multirow[t]{2}{*}{$2-34 \%[13]$} & Erythema nodosum & & Yes [25] \\
\hline & & Pyoderma gangrenosum & & No [25] \\
\hline & $13 \%[20]$ & Hidradenitis suppurativa & & Yes [20] \\
\hline \multirow[t]{2}{*}{ Ocular } & \multirow[t]{2}{*}{$0.3-5 \%[13]$} & Episcleritis & & Yes [25] \\
\hline & & Uveitis & & Case dependent [28] \\
\hline \multirow[t]{3}{*}{ Hepatopancreatobiliary } & $30 \%[11]$ & Abnormal liver chemistry & & Case dependent [11] \\
\hline & $13-34 \%[13]$ & Gallstones & & No [11] \\
\hline & $2-5 \%[13]$ & $\begin{array}{l}\text { Primary sclerosing cholangitis } \\
\text { Bile duct carcinoma } \\
\text { Drug-induced liver injury } \\
\text { Pancreatitis }\end{array}$ & & Case dependent [25] \\
\hline Metabolic & $20-50 \%[11]$ & Low bone mass/osteoporosis & & Case dependent [11] \\
\hline Renal & $6-23 \%[13]$ & Kidney stones & & Case dependent [13] \\
\hline Hematologic & $1.4-3.3 \%[11]$ & Thromboembolism & & Yes [11] \\
\hline
\end{tabular}


and for other musculoskeletal EIMs that are refractory to nonsteroidal anti-inflammatory drugs [11], only a few studies have investigated the use of these agents in IBD-related arthritides. A sub-analysis of the CHARM trial, including $420 \mathrm{CD}$ patients with arthritis and arthralgia symptoms at baseline, showed that 70 of the 281 patients (24.9\%) treated with adalimumab were free of arthritis and arthralgia after 56 weeks of therapy compared with 11 of the 139 patients (7.9\%) treated with placebo [18].

Dermatologic EIMs include a broad spectrum of skin diseases, and it is not uncommon for patients to develop multiple manifestations concurrently during the natural course of IBD. Two major immune-mediated forms of dermatologic EIMs are erythema nodosum (EN) and pyoderma gangrenosum (PG) [19]. In addition, hidradenitis suppurativa affects about $13 \%$ of IBD patients and is characterized by painful, inflamed skin follicles commonly found in the axillae, inguinal, and anogenital areas [20]. Psoriasis is another skin disease that is common among IBD patients. The prevalence of psoriasis in the general population is approximately $1.5 \%$ [21], and patients with IBD have a sixfold risk of developing the disease [22]. As is the case for spondyloarthropathies, TNF antagonists are highly effective therapy for psoriasis. However, some patients develop psoriasiform lesions, typically on the scalp, hands, and feet following prolonged treatment with these drugs. In many cases, treatment requires withdrawal of the TNF antagonist and switching to another class of agent, typically ustekinumab or vedolizumab.

EN, which affects $15 \%$ of $\mathrm{CD}$ and $10 \%$ of UC patients [23], is a small vessel vasculitis that results in panniculitis. The lesions typically present as inflamed nodules of $1 \mathrm{~cm}$ to $5 \mathrm{~cm}$ in diameter on the lower limbs and occasionally on the arms and trunk [23]. Treatment of EN usually consists of corticosteroids and intensification of medical therapy to control intestinal disease. A strong correlation exists between the presence of EN and intestinal disease activity.

PG is less common, occurring in $0.4-2 \%$ of IBD patients, and is usually more severe than EN [24]. Pathologically, PG is characterized by full thickness necrosis of the skin due to vasculitis. The disease manifests as nodules that evolve into deep ulcerations typically on the shins but can develop anywhere on the skin [23]. PG is more common in UC than CD. Unlike EN, PG is normally independent of luminal IBD disease activity [25]. Although ECCO guidelines suggest corticosteroids as a first-line therapy for PG, severe cases require treatment with immunosuppressives such as azathioprine, TNF antagonists, or calcineurin inhibitors [11]. A small $(n=13)$ randomized controlled trial for the treatment of PG showed 6 (46\%) of the infliximab treated patients improved compared with only 1 of 17 (6\%) patients assigned to placebo [26].

Ocular complications include episcleritis and uveitis and are often present concurrently with other EIMs [27].
Episcleritis, which is more common in $\mathrm{CD}$ than in UC, often correlates with intestinal disease activity [25] and presents with hyperemia, irritation, burning, and tenderness [28]. Uveitis is less common than episcleritis, and the association with IBD activity is variable. Patients with uveitis present with ocular pain, blurred vision, photophobia, and headaches [28]. While some cases of episcleritis are self-limiting, the risk of blindness associated with uveitis mandates ophthalmologic referral and prompt initiation of therapy [28]. ECCO guidelines currently recommend topical corticosteroids for symptomatic treatment and systemic corticosteroids or immunosuppressives, including biologics, in severe or resistant cases [11, 29].

\section{Vedolizumab for the Treatment of EIMs}

Vedolizumab is unique among biologics used to treat IBD because it specifically targets the gastrointestinal tract [30]. Vedolizumab binds to the $\alpha 4 \beta 7$ integrin that is expressed on activated gut-homing $\mathrm{T}$ lymphocytes, and blocks the interaction of $\alpha 4 \beta 7$ and mucosal addressin cell adhesion molecule 1 (MAdCAM-1) [31]. MAdCAM-1 is preferentially expressed on the endothelium of blood vessels in the gastrointestinal tract. The gastrointestinal tract-specific interaction between $\alpha 4 \beta 7$ integrins and MAdCAM- 1 allows for gut-targeted therapy, thereby likely reducing side effects associated with systemic immunosuppression.

The gut-selective mode of action of vedolizumab has demonstrated efficacy in the treatment of UC and CD in the phase 3 GEMINI 1 [32] and GEMINI 2 [33] studies, respectively. An integrated summary of 6 phase 2 and phase 3 vedolizumab trials also provided evidence for the safety of vedolizumab, which included a low incidence of the infectious side effects that are typically associated with non-selective treatments [34].

Although the GEMINI studies were not powered to assess EIMs, post hoc analyses of the GEMINI 2 CD data indicated a potential benefit with vedolizumab in resolving symptoms of arthralgias. In these analyses, EIMs were evaluated post hoc and defined by the CD Activity Index (CDAI) complications component as the presence of arthritis/arthralgia, iritis/uveitis, EN, PG, aphthous stomatitis, or fever. Resolution of EIMs was defined by the absence of CDAI complications at each post-baseline study visit during the 52-week study [35]. Kaplan-Meier estimates for the resolution of these events were $18 \%$ at week 26 and $43 \%$ at week 52 in vedolizumab-treated patients (compared with 4 and $23 \%$ in those assigned to placebo, respectively) with a hazard ratio of 1.84 (95\% confidence interval, 0.91-3.71) [35]. Analysis of these data also revealed that patients who received vedolizumab were $32 \%$ more likely to achieve sustained resolution of 
Table 2 Cases

\begin{tabular}{|c|c|c|c|c|c|c|}
\hline & Age & IBD & EIM & Correlates with IBD? & $\begin{array}{l}\text { Concomitant } \\
\text { medications }\end{array}$ & Outcome of vedolizumab treatment \\
\hline Case 1 & 50 & $\mathrm{CD}$ & Ankylosing spondylitis and sacroiliitis & No & None & $\begin{array}{l}\text { Resolved } 5 \text { months into vedolizumab treat- } \\
\text { ment }\end{array}$ \\
\hline Case 2 & 44 & $\mathrm{CD}$ & Polyarthritis (back and knees) & Yes & Budesonide & $\begin{array}{l}\text { Resolved } 4 \text { months into vedolizumab treat- } \\
\text { ment } \\
2 \text { years into vedolizumab treatment, knee } \\
\text { pain remains resolved, back pain has } \\
\text { returned }\end{array}$ \\
\hline Case 3 & 34 & UC & Joint and back pain & Yes & None & $\begin{array}{l}\text { Resolved } 1 \text { month into vedolizumab treat- } \\
\text { ment }\end{array}$ \\
\hline Case 4 & 18 & UC & Polyarticular arthropathy & No & Mesalamine & Resolved after third dose of vedolizumab \\
\hline Case 5 & 46 & $\mathrm{CD}$ & EN & Yes & Mesalamine & Resolved after third dose of vedolizumab \\
\hline Case 6 & 24 & $\mathrm{UC}$ & PG & No & None & Resolved after sixth dose of vedolizumab \\
\hline Case 7 & 87 & $\mathrm{CD}$ & PG & No & Methotrexate & $\begin{array}{l}\text { PG improved but not resolved after } 6 \text { months } \\
\text { PG resolved after } 4 \text { months of increasing } \\
\text { infusion frequency to every } 4 \text { weeks } \\
\text { Recurrent PG } 9 \text { months after increasing infu- } \\
\text { sion frequency } \\
\text { PG at pre-vedolizumab severity } 12 \text { months } \\
\text { after increasing infusion frequency } \\
\text { Vedolizumab discontinued, PG resolved after } \\
\text { treatment with ustekinumab }\end{array}$ \\
\hline Case 8 & 24 & $\mathrm{UC}$ & Uveitis & Case dependent & Mesalamine & Resolved after third dose of vedolizumab \\
\hline
\end{tabular}

$C D$ Crohn's disease, EIM extraintestinal manifestation, $E N$ erythema nodosum, $I B D$ inflammatory bowel disease, $P G$ pyoderma gangrenosum, $U C$ ulcerative colitis

arthritis/arthralgia and $21 \%$ less likely to have a worsening or new occurrence than those assigned to placebo [36]; however, few descriptions regarding the efficacy of vedolizumab on EIMs based on real-world clinical experience have been published.

Herein, we present 8 cases describing our clinical experience with vedolizumab for the treatment of IBD patients who present with various EIMs including peripheral arthralgia/arthritis, axial arthropathies, PG, EN, and uveitis (Table 2).

\section{Cases}

\section{Case 1}

A 50-year-old male with CD diagnosed in 1992 developed ankylosing spondylitis and sacroiliitis in 2002 . The sacroiliitis presented with edema in the lowest sacral segment and the highest coccyx segment (Fig. 1). Prior treatment consisted of infliximab from 2002 to 2014, prednisolone for 2 months between 2012 and 2013, and azathioprine from 2007 to 2014. Infliximab and azathioprine were discontinued in 2014 because of recurrent peritonsillar abscesses, infectious keratoconjunctivitis, acne causing skin abscesses, and failure to achieve symptomatic remission. The patient then initiated treatment with vedolizumab. At the time, the patient had 3-4 bowel movements per day with some mucus, a fecal calprotectin concentration of $722 \mathrm{mg} / \mathrm{kg}$, and primary symptoms of back pain, stiffness, and joint pain. Five months after initiation of treatment with vedolizumab, these symptoms resolved, and the patient had 1 bowel movement per day without mucus and a fecal calprotectin concentration that had decreased to $33 \mathrm{mg} / \mathrm{kg}$. Overall, CD symptoms improved and were well controlled throughout vedolizumab treatment. One year after resolution of symptoms, the patient experienced acne-like skin lesions on the face, which were diagnosed as rosacea that immediately resolved with tetracycline treatment.

\section{Case 2}

A 44-year-old male with CD initially diagnosed in 2009 developed polyarthritis in 2010. He had also been experiencing back pain since 1991. Prior treatments included prednisolone for 2 months in 2009 and 1 month in 2010, budesonide for 1 month in 2009 and 2 months in 2015, methotrexate for 2 months in 2010 (discontinued because of diffuse skin rash), azathioprine for 1 week in 2009 (discontinued because of pancreatitis), adalimumab for 1 month in 


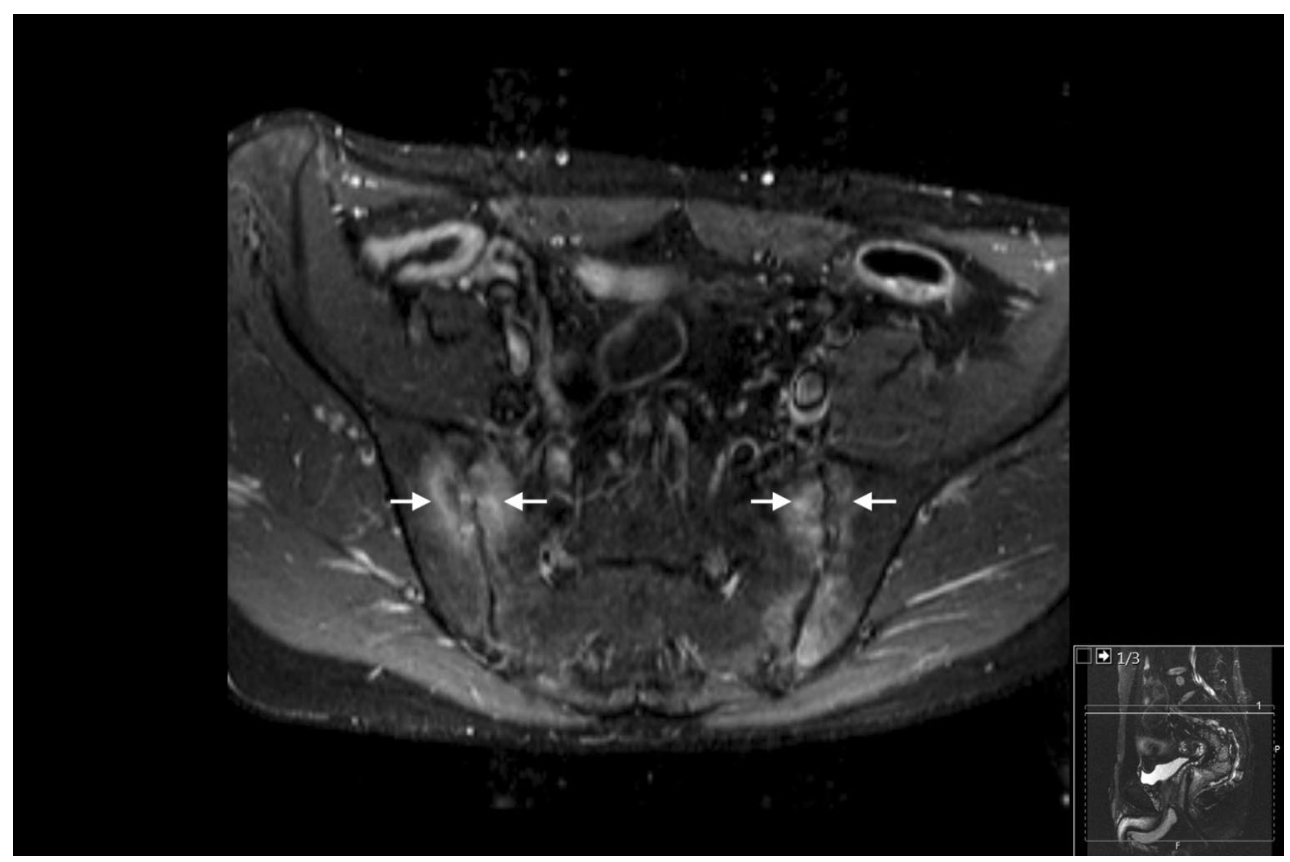

Fig. 1 MRI findings of the 50-year-old male in case 1 before treatment. Arrows in the axial MR image of the sacroiliac joints indicate articular irregularities and periarticular edema as signs of sacroiliitis

2010 (discontinued because of swollen and painful joints), sulfasalazine for 1 month in 2011 (discontinued because of lack of response), and natalizumab from 2011 to 2014.

Natalizumab was effective in controlling both the luminal symptoms and the polyarthritis; however, it was discontinued for safety concerns because the patient was positive for antibodies to the John Cunningham (JC) virus. After discontinuation, the patient presented with back pain likely caused by degenerative changes and knee pain from arthritis. Intestinal symptoms included $4-5$ bowel movements per day with a small amount of blood and mucus and moderate abdominal pain. Vedolizumab treatment was initiated in 2014, and 4 months later all of his arthritic complaints had resolved. Although some back pain returned 2 years after initiation of treatment, the patient's knee pain remained resolved. Furthermore, the patient's stool frequency had improved to 2 loose stools per day with no blood or abdominal pain. It is noteworthy that this patient developed a perianal abscess that was drained in 2015 and a perianal fistula that was resected in 2016. It is unclear whether a causal relationship exists between vedolizumab and these events.

\section{Case 3}

A 34-year-old male with UC diagnosed in 2012 presented with joint and back pain in 2013. The pain mainly affected his back; however, he also had peripheral arthritis type 1 with severe pain in his hips, knees, and muscles. No diagnosis of sacroiliitis was confirmed despite MR imaging of the lumbosacral spine and pelvis. Prior treatments included prednisolone for 2 months in 2013, mesalamine for 1 week in 2013 (discontinued because of myocarditis), infliximab for 14 months between 2013 and 2014 (discontinued because of lack of efficacy and development of antidrug antibodies), and golimumab for 7 months between 2014 and 2015 (discontinued because of insomnia and migraine).

Vedolizumab treatment was initiated in 2015, and after 1 month the patient's back and joint pain resolved. However, for the first 5 months he received vedolizumab, the patient experienced arthralgias in both his back and peripheral joints approximately 5 days before the vedolizumab infusions. One year after resolution of symptoms, the patient developed pityriasis rosea on his abdomen, which resolved spontaneously within 2 months without treatment. The patient continues to be in remission on vedolizumab therapy.

\section{Case 4}

An 18-year-old female with UC diagnosed in 2014 had polyarticular arthropathy diagnosed in 2016. At the time of presentation, the patient was being treated with mesalamine and was naïve to corticosteroids.

Although a definitive diagnosis of UC was not made until 2016, the patient had been experiencing arthralgias in her hands and knees since her initial presentation. Colonoscopy showed no active disease, and her C-reactive protein (CRP) and fecal calprotectin concentrations were normal; however, random colon biopsies revealed mildly active 
histopathologic disease. Although the entire colon appeared to be normal on endoscopic examination, histological evaluation revealed mild-to-moderate active disease. Characteristics of mild-to-moderate chronic disease, including cryptitis, crypt abscesses, mild-to-moderate mucosal ulceration, and moderate crypt architectural distortion, were observed. The patient opted to initiate vedolizumab after being offered the options of treatment with corticosteroid, immunosuppressives, or TNF antagonists. The patient noted that the joint pains resolved completely after the third induction dose of vedolizumab.

\section{Case 5}

A 46-year-old female with CD diagnosed in 2005 received multiple courses of corticosteroid therapy resulting in initiation of azathioprine. However, this agent was discontinued because pancreatitis developed within 2 weeks of treatment. Although infliximab monotherapy was started, it was not possible to provide continuous treatment for insurance reasons. In 2015, the patient experienced a rash and shortness of breath following re-initiation of infliximab and the medication was discontinued.

In 2016, the patient presented with right lower quadrant pain and increasingly frequent non-bloody stools. Physical examination revealed right lower quadrant tenderness and a questionable mass. Tender, raised red nodules of EN were present on the extensor surfaces of both tibias. Her white blood cell (WBC) count was $14.6 \times 10^{9} / \mathrm{L}$, erythrocyte sedminentation rate (ESR) was $34 \mathrm{~mm} / \mathrm{h}$, and the serum CRP concentration was $6 \mathrm{mg} / \mathrm{L}$. Stool calprotectin concentrations were elevated. Magnetic resonance enterography showed active inflammation of the terminal ileum. A colonoscopy to the terminal ileum revealed a normal colon examination, but multiple ulcers were present in the terminal ileum. Biopsies from the ileum were consistent with active $\mathrm{CD}$, with a single non-caseating granuloma demonstrated.

Treatment with vedolizumab was initiated, and resolution of the skin lesions followed the third infusion of the drug. The abdominal pain resolved completely after the fifth dose. The CRP, ESR, and stool calprotectin values were also normal after the fifth dose. The patient continued treatment with vedolizumab, and mesalamine was discontinued. Repeat endoscopy revealed a normal colon and ileum; however, the ileocecal valve was noted to be deformed and mildly stenotic. Biopsies of the colon and valve were normal, and biopsies of the ileum revealed mild enteritis.

\section{Case 6}

A 24-year-old male with comorbid osteoporosis and diabetes, who was diagnosed with UC in 1998, presented with PG in 2016. Prior treatments were 6-mercaptopurine, mesalamine, corticosteroids, and multiple TNF antagonists. The patient underwent a colectomy and permanent ileostomy in 2012.

In 2016, the patient presented with a peristomal ulceration. The lesion had irregular borders and was diagnosed by a dermatologist as PG. The patient was positive for anti-saccharomyces cerevisiae antibodies (ASCA) and anti-neutrophil cytoplasmic antibodies (ANCA). The patient deferred corticosteroid treatment because of his osteoporosis. Small bowel was normal by endoscopy and video capsule endoscopy. The patient began vedolizumab therapy in 2016, and the lesion resolved after the sixth dose. The patient remains on vedolizumab and has been lesion free for 1 year.

\section{Case 7}

An 87-year-old patient with a history of CD underwent an end ileostomy in 1972, and in 2014, he developed peristomal PG. The patient was treatment naïve since surgery, CD symptoms were well controlled, and luminal disease was not present at the time of PG presentation. Treatment with infliximab and methotrexate was commenced in 2015, leading to improvement in PG. However, 6 months after initiation of treatment, the patient developed a diffuse rash and infliximab was discontinued. Subsequently, the peristomal ulcer worsened. After a period without any biologic therapy, the patient began treatment with vedolizumab and continued methotrexate. Six months later, the PG had improved but was not resolved. Although PG improved with maintenance infusions dosed every 8 weeks, it began to worsen 4-5 weeks after each infusion. In response, treatment was intensified to an infusion every 4 weeks, which led to complete healing of PG after 4 months.

Nine months into the adjusted vedolizumab infusion schedule, the patient began to experience recurrent PG. Three months later, PG worsened to the severity level exhibited before the initiation of vedolizumab. The patient discontinued vedolizumab and began treatment with ustekinumab in 2017 and achieved complete healing.

\section{Case 8}

A 24-year-old male with UC diagnosed in 2014, following presentation with bloody diarrhea that required hospitalization, developed uveitis in 2016. After intravenous corticosteroid induction therapy, the patient received mesalamine for maintenance or remission, which was successful for 2 years. In 2016, the patient presented with headaches, bilateral ocular pain, and blurred vision without symptoms of bloody diarrhea or abdominal cramps. Although the stool fecal calprotectin concentration was within the normal range, the CRP concentration was $14 \mathrm{mg} / \mathrm{L}$ and ESR was $64 \mathrm{~mm} / \mathrm{h}$. An initial colonoscopy revealed moderately active pancolitis, and 
biopsies confirmed the endoscopic findings. The patient's ophthalmologic assessment revealed anterior chamber uveitis. Treatment with oral prednisone $40 \mathrm{mg}$ daily for 2 weeks was initiated with tapering by $5 \mathrm{mg}$ weekly. The patient developed recurrent symptoms when the prednisone dose was reduced to $15 \mathrm{mg}$. At this time, a colonoscopy revealed mild disease of the rectum and sigmoid, which was confirmed by biopsies.

Subsequently, treatment with vedolizumab was initiated. Corticosteroid tapering was resumed after the third dose of vedolizumab. The patient had no recurrence of uveitis and was off prednisone 1 year later, when a repeat colonoscopy showed a normal mucosa. Biopsies confirmed the absence of active histopathologic inflammation.

\section{Discussion}

The occurrence of EIMs with IBD is an important challenge for clinicians who treat IBD. These entities are relatively common and contribute substantially to the burden of illness of both CD and UC. Very little high-quality research has been conducted into the pathogenesis, epidemiology, or therapy of these conditions. Consequently, many important gaps exist in our approach to the management of EIMs. Although conventional wisdom is that some of the individual EIMs, such as arthralgias, track closely with the presence of luminal disease activity, whereas others, such as sacroiliitis, follow an independent course, this dogma can be challenged.

In keeping with this notion, some of the cases presented in this paper describe patients with quiescent gastrointestinal tract disease and continuing symptoms of arthralgias. Alternatively, cases of uveitis and sacroiliitis are depicted that resolve with more intense management of intestinal disease. Even though these cases cannot be considered strong scientific evidence to refute the established relationships between EIMs and disease activity, the classic studies that have previously evaluated this question did not rigorously evaluate patients' luminal disease activity by endoscopy, nor did they assess EIMs using objective or qualitative measures. These cases are valuable in that they underscore the need to further examine the relationship between intestinal disease activity and EIM severity using rigorous methodologies.

TNF antagonists are thought to be effective for the treatment of EIMs, and a systematic review that included 9 interventional, 7 open-label, and 13 noninterventional studies concluded that they are beneficial for the treatment of musculoskeletal, cutaneous, and ocular manifestations [37]. Data from the CARE study, a large multicenter openlabel phase 3 trial in patients with moderately to severely active $C D$, show that after 20 weeks of adalimumab treatment in 497 patients with baseline EIMs, 79\% had resolution of at least one EIM and 51\% were free of EIM signs and symptoms [38]. A multinational phase 3 trial has also shown that adalimumab is effective in treating idiopathic uveitis by reducing both inflammation and visual impairment [13]. For PG, specifically, infliximab demonstrated complete healing of skin lesions in a retrospective study of 13 patients with IBD and medically refractory PG; 3 patients had a complete response to only induction infliximab therapy, while 10 patients required infusions every 4-12 weeks for maintained PG healing [26, 39].

Although TNF antagonists are beneficial for the management of EIMs, these agents are not uniformly effective, are poorly tolerated in some patients, and frequently become ineffective owing to sensitization or other poorly defined mechanisms. Our observations here suggest that vedolizumab may be an effective treatment option for the management of EIMs, especially those that track closely with luminal disease. In this case series, vedolizumab therapy was effective for PG in UC, uveitis, EN, polyarticular arthropathy, and ankylosing spondylitis/sacroiliitis, but did not provide sustained benefit for the treatment of PG in CD. Notably, most of these patients had good control of their luminal IBD, which suggests that control of intestinal inflammation may be critical for the management of EIMs. The efficacy of vedolizumab in treating EIMs that are known to correlate with disease is not surprising, as analysis of the GEMINI 2 clinical trial data showed that patients treated with vedolizumab were $32 \%$ more likely to achieve sustained resolution of arthritis/arthralgia compared with placebo [40]. However, the cases described herein of patients with EIMs that were traditionally thought to be independent of bowel disease, such as PG and axial spondyloarthritis ( $\mathrm{SpA})$, are interesting. These results are consistent with a recent study conducted by Orlando and colleagues [41] in 53 patients with IBD-associated SpA, the majority of whom had failed to respond to a TNF antagonist and were corticosteroid dependent. Of the 14 patients with active SpA in this study, 6 patients demonstrated a strong clinical benefit after initiation of vedolizumab treatment. In another observational study, a correlation was shown between an improvement in symptoms and decrease in MR-defined bone marrow edema and gut inflammation in patients with active SpA [42].

In summary, the findings of these cases highlight the need to further examine the relationship between intestinal disease activity and EIMs and suggest that vedolizumab has a role in the management of these patients. Given the limited data available, large-scale cohort studies and randomized controlled trials that employ objective measures of inflammation are needed before these questions can be answered definitively.

Acknowledgments Medical writing assistance was provided by Michael D'Ecclessis, Ph.D., and Martina Schwarzkopf, Ph.D., of inVentiv Medical Communications and funded by Takeda Pharmaceuticals U.S.A., Inc. 


\section{Compliance with ethical standards}

Conflict of interest Dr. Feagan has received grant support from $\mathrm{Ab}$ bVie, Amgen, AstraZeneca, Bristol-Myers Squibb, Roche, Genentech, J\&J, Janssen, Millennium, Pfizer, Receptos, Tillotts, and UCB and served as a consultant or advisory board member for AbbVie, ActoGeniX, Akros, Albireo, Amgen, AstraZeneca, Avaxia Biologics, Avir Pharma, Baxter Healthcare Corp, Biogen Idec, Boehringer Ingelheim, Bristol-Myers Squibb, Calypso Biotech, Celgene, Elan/ Biogen, enGene, Ferring Pharmaceuticals, Galapagos, Genentech/ Roche, GiCare Pharma, Gilead, Given Imaging, GSK, Inception IBD Inc, Ironwood Pharmaceuticals, J\&J, Janssen, Japan Tobacco, Kyowa Hakko Kirin Co Ltd, Lexicon, Lilly, Lycera Biotech, Merck, Mesoblast Ltd, Millennium, Nektar, Nestlé, Novartis, Novo Nordisk, Pfizer, Prometheus Therapeutics \& Diagnostics, Protagonist, Receptos, Salix, Shire, Sigmoid Pharma, Synergy Pharmaceuticals Inc, Takeda, Teva Pharmaceutical Industries Ltd, TiGenix, Tillotts, UCB Pharma, Vertex Pharmaceuticals, VHsquared Ltd, Warner Chilcott, Wyeth, Zealand Pharma, and Zyngenia. Dr. Marsal has received grants from the Healthcare Region of Southern Sweden, Skane University Hospital, and the Swedish government; grants and personal fees from Abbvie, Ferring, Hospira; and personal fees from Bristol-Myers Squibb, Takeda and Tillotts. Dr. Lee has received grant and research support from AbbVie, Arena, Atlantic, Celgene, Gilead Sciences, Janssen, Pfizer, Salix, Shield, Takeda, Tetherex, UCB Pharma and has performed consulting for Arena, Celgene, Celltrion Healthcare, Cornerstones, Eli Lilly and Company, Janssen, Mesoblast, Pfizer, Salix, Takeda, and UCB Pharma. Dr. Fleisher has served on the speaker bureau for Janssen and Takeda. Drs. Korelitz, Frado, and Parian have nothing to disclose.

Informed consent A written informed consent was obtained from patient cases 1, 2, 3, 4, 5, 6, and 8. Case 7 was de-identified and was in compliance with University of Washington Medical Center's IRB, which does not require an informed consent for single case reports of de-identified patients.

Open Access This article is distributed under the terms of the Creative Commons Attribution-NonCommercial 4.0 International License (http://creativecommons.org/licenses/by-nc/4.0/), which permits any noncommercial use, distribution, and reproduction in any medium, provided you give appropriate credit to the original author(s) and the source, provide a link to the Creative Commons license, and indicate if changes were made.

\section{References}

1. Dulai PS, Singh S, Casteele NV, Boland BS, Sandborn WJ. How will evolving future therapies and strategies change how we position the use of biologics in moderate to severely active inflammatory bowel disease. Inflamm Bowel Dis. 2016;22:998-1009.

2. Silva FA, Rodrigues BL, Ayrizono ML, Leal RF. The immunological basis of inflammatory bowel disease. Gastroenterol Res Pract. 2016;2016:2097274.

3. Colombel JF, Narula N, Peyrin-Biroulet L. Management strategies to improve outcomes of patients with inflammatory bowel diseases. Gastroenterology. 2017;152(351-361):e355.

4. Frolkis AD, Dykeman J, Negron ME, et al. Risk of surgery for inflammatory bowel diseases has decreased over time: a systematic review and meta-analysis of population-based studies. Gastroenterology. 2013;145:996-1006.
5. Moss AC. Optimizing the use of biological therapy in patients with inflammatory bowel disease. Gastroenterol Rep (Oxf). 2015;3:63-68.

6. Colombel JF, Sandborn WJ, Reinisch W, et al. Infliximab, azathioprine, or combination therapy for Crohn's disease. $N$ Engl J Med. 2010;362:1383-1395.

7. Colombel JF, Rutgeerts PJ, Sandborn WJ, et al. Adalimumab induces deep remission in patients with Crohn's disease. Clin Gastroenterol Hepatol. 2014;12:414-422.e5.

8. Schreiber S, Reinisch W, Colombel JF, et al. P178 early Crohn's disease shows high levels of remission to therapy with adalimumab: sub-analysis of CHARM. J Crohn's Colitis Suppl. 2007; $1: 49$

9. Peyrin-Biroulet L, Sandborn W, Sands BE, et al. Selecting therapeutic targets in inflammatory bowel disease (STRIDE): Determining therapeutic goals for treat-to-target. Am J Gastroenterol. 2015;110:1324-1338.

10. Lakatos PL, Lakatos L, Kiss LS, Peyrin-Biroulet L, Schoepfer A, Vavricka S. Treatment of extraintestinal manifestations in inflammatory bowel disease. Digestion. 2012;86(Suppl 1):28-35.

11. Harbord M, Annese V, Vavricka SR, et al. The first European evidence-based consensus on extra-intestinal manifestations in inflammatory bowel disease. J Crohns Colitis. 2016;10:239-254.

12. Bhagat S, Das KM. A shared and unique peptide in the human colon, eye, and joint detected by a monoclonal antibody. Gastroenterology. 1994;107:103-108.

13. Levine JS, Burakoff R. Extraintestinal manifestations of inflammatory bowel disease. Gastroenterol Hepatol (NY). 2011;7:235-241.

14. Orchard TR, Wordsworth BP, Jewell DP. Peripheral arthropathies in inflammatory bowel disease: their articular distribution and natural history. Gut. 1998;42:387-391.

15. Voulgari PV. Rheumatological manifestations in inflammatory bowel disease. Ann Gastroenterol. 2011;24:173-180.

16. Atzeni F, Ardizzone S, Bertani L, Antivalle M, Batticciotto A, Sarzi-Puttini P. Combined therapeutic approach: inflammatory bowel diseases and peripheral or axial arthritis. World J Gastroenterol. 2009;15:2469-2471.

17. van der Heijde D, Kivitz A, Schiff MH, et al. Efficacy and safety of adalimumab in patients with ankylosing spondylitis: results of a multicenter, randomized, double-blind, placebo-controlled trial. Arthritis Rheum. 2006;54:2136-2146.

18. Schwartz D, Lofberg R, Pollack P. Adalimumab-treated patients with moderate to severe Crohn's disease experienced reductions in extraintestinal manifestations data from CHARM. Am J Gastroenterol. 2009;104:S465.

19. Zippi M, Pica R, De Nitto D, Paoluzi P. Biological therapy for dermatological manifestations of inflammatory bowel disease. World J Clin Cases. 2013;1:74-78.

20. Principi M, Cassano N, Contaldo A, et al. Hydradenitis suppurativa and inflammatory bowel disease: an unusual, but existing association. World J Gastroenterol. 2016;22:4802-4811.

21. Nevitt GJ, Hutchinson PE. Psoriasis in the community: prevalence, severity and patients' beliefs and attitudes towards the disease. Br J Dermatol. 1996;135:533-537.

22. Stolwijk C, van Tubergen A, Castillo-Ortiz JD, Boonen A. Prevalence of extra-articular manifestations in patients with ankylosing spondylitis: a systematic review and meta-analysis. Ann Rheum Dis. 2015;74:65-73.

23. Greenstein AJ, Janowitz HD, Sachar DB. The extra-intestinal complications of Crohn's disease and ulcerative colitis: a study of 700 patients. Medicine (Baltimore). 1976;55:401-412.

24. Bernstein CN, Blanchard JF, Rawsthorne P, Yu N. The prevalence of extraintestinal diseases in inflammatory bowel disease: a population-based study. Am J Gastroenterol. 2001;96:1116-1122. 
25. Vavricka SR, Schoepfer A, Scharl M, Lakatos PL, Navarini A, Rogler G. Extraintestinal manifestations of inflammatory bowel disease. Inflamm Bowel Dis. 2015;21:1982-1992.

26. Brooklyn TN, Dunnill MG, Shetty A, et al. Infliximab for the treatment of pyoderma gangrenosum: a randomised, double blind, placebo controlled trial. Gut. 2006;55:505-509.

27. Korelitz BI, Coles RS. Uveitis (iritis) associated with ulcerative and granulomatous colitis. Gastroenterology. 1967;52:78-82.

28. Rothfuss KS, Stange EF, Herrlinger KR. Extraintestinal manifestations and complications in inflammatory bowel diseases. World $J$ Gastroenterol. 2006;12:4819-4831.

29. Jaffe GJ, Dick AD, Brezin AP, et al. Adalimumab in patients with active noninfectious uveitis. N Engl J Med. 2016;375:932-943.

30. Wyant T, Fedyk E, Abhyankar B. An overview of the mechanism of action of the monoclonal antibody vedolizumab. J Crohns Colitis. 2016;10:1437-1444.

31. Pijls PA, Gilissen LP. Vedolizumab is an effective alternative in inflammatory bowel disease patients with anti-TNF-alpha therapy-induced dermatological side effects. Dig Liver Dis. 2016;48:1391-1393.

32. Feagan BG, Rutgeerts P, Sands BE, et al. Vedolizumab as induction and maintenance therapy for ulcerative colitis. $N$ Engl J Med. 2013;369:699-710.

33. Sandborn WJ, Feagan BG, Rutgeerts $\mathrm{P}$, et al. Vedolizumab as induction and maintenance therapy for Crohn's disease. $N$ Engl J Med. 2013;369:711-721.

34. Colombel JF, Sands BE, Rutgeerts P, et al. The safety of vedolizumab for ulcerative colitis and Crohn's disease. Gut. 2017;66:839-851.

35. Rubin D, Feagan B, Dryden G, et al. The effect of vedolizumab on extraintestinal manifestations in patients with Crohn's disease in GEMINI 2. Crohn's Colitis Found Am. 2016;22:S42-S43.
36. Feagan BG, Rubin DT, Danese S, et al. Efficacy of vedolizumab induction and maintenance therapy in patients with ulcerative colitis, regardless of prior exposure to tumor necrosis factor antagonists. Clin Gastroenterol Hepatol. 2017;15:229-239.e5.

37. Peyrin-Biroulet L, Van Assche G, Gomez-Ulloa D, et al. Systematic review of tumor necrosis factor antagonists in extraintestinal manifestations in inflammatory bowel disease. Clin Gastroenterol Hepatol. 2017;15:25-36.e27.

38. Lofberg R, Louis EV, Reinisch W, et al. Adalimumab produces clinical remission and reduces extraintestinal manifestations in Crohn's disease: results from CARE. Inflamm Bowel Dis. 2012;18:1-9.

39. Regueiro M, Valentine J, Plevy S, Fleisher MR, Lichtenstein GR. Infliximab for treatment of pyoderma gangrenosum associated with inflammatory bowel disease. Am J Gastroenterol. 2003;98:1821-1826.

40. Feagan BG, Sandborn WJ, Colombel JF, et al. DOP019 Effect of vedolizumab treatment on extraintestinal manifestations in patients with Crohn's disease: a GEMINI 2 post hoc analysis. Gastroenterology. 2017;152:S597.

41. Orlando A, Orlando R, Ciccia F, et al. Clinical benefit of vedolizumab on articular manifestations in patients with active spondyloarthritis associated with inflammatory bowel disease. Ann Rheum Dis. 2017;76:e31.

42. Van Praet L, Jans L, Carron P, et al. Degree of bone marrow oedema in sacroiliac joints of patients with axial spondyloarthritis is linked to gut inflammation and male sex: results from the GIANT cohort. Ann Rheum Dis. 2014;73:1186-1189. 